\title{
Das Jahr der Hacks
}

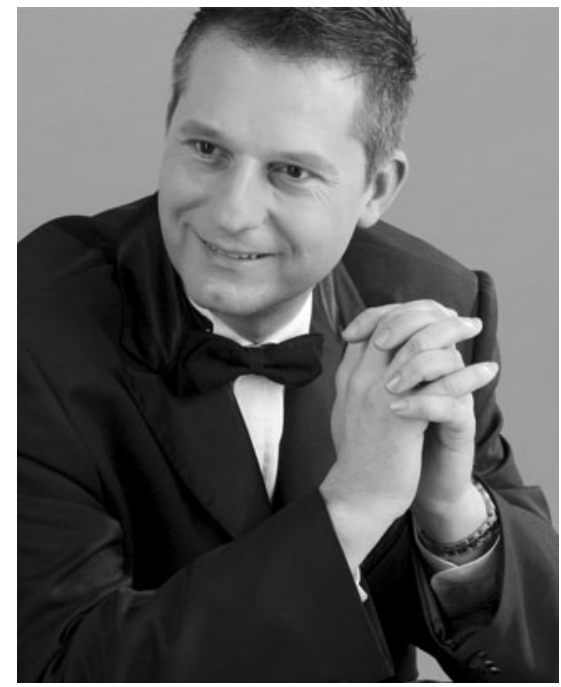

Unlängst bezeichnete ein international anerkannter IT Experte das vergangene Jahr als das „Jahr der Hacks!“, was er auf die zahlreichen populären Internet Angriffe bezog. In der Tat kann man versuchen, eine Liste aller dieser Angriffe in 2011 auf personenbezogenen Daten und Kreditkartendaten zu erstellen, und wird schnell im dreistelligen Bereich landen.

Was haben wir verpasst? Was ist passiert seit legendäre Experten wie Lipner und McGraw (siehe Artikel im Heft) vor über 10 Jahren einen „Secure Development Lifecycle“ entwickelt haben? Softwareunternehmen tätigen immense Investitionen in Softwaresicherheit; das Thema hat inzwischen Einzug in Curricula der Informatikausbildung gefunden; und es gibt gemeinnützige Organisationen wie „SAFECode", die das Know-how der großen IT Unternehmen weiter verbreiten.

Ein neuer Faktor fügt sich nun in dieses Spannungsfeld ein: „Cyberwar“ - Angriffe auf IT Systeme, vorbereitet und durchgeführt von staatlichen Organisationen mit entsprechenden Budgets für professionelle Angriffe.

Während es vor 10 Jahren nur wenige „Hacker“ mit wirklich professionellen Fähigkeiten gab, die kaum Schaden anrichteten sondern lediglich für Ruhm und Ehre arbeiteten, hat sich die Bedrohungslage nicht zuletzt durch die "Cyberwar" Aktivitäten und neue Aktivistengruppen wie „Anonymous" grundlegend gewandelt.

Auf der Gesetzgebungsseite kündigen sich massive Änderungen mit der Europäischen Datenschutzgrundverordnung an, aus der sich auch Sicherheitsanforderungen für die Softwareentwicklung ableiten werden. Datenschutz ohne Software Sicherheit ist schlichtweg nicht umsetzbar. In den USA beschäftigt sich eine Vielzahl von Regierungsgremien mit „Supply Chain Security". In Einkaufsrichtlinien werden Sicherheitsanforderungen an staatlich genutzte Software oder Softwaremodule festgeschrieben. Der Ruf nach Zertifizierung (z.B.,,Common (riteria") wird immer lauter, obwohl den Beteiligten auch klar ist, dass ein vollständiger Zertifizierungszwang die Innovationsgeschwindigkeit deutlich herabsetzen wird.

Nach wie vor tut sich die Gesetzgebung sehr schwer mit internationaler Strafverfolgung von Cyber-Kriminalität. Es gibt eine Reihe von Erfolgen: Microsoft engagiert sich z.B. erfolgreich im Abschalten von Bot-Netzen. Der Großteil der "Dropzones“, die gestohlene Kreditkarten und andere vertrauliche Daten sammeln, entgehen aber dem Zugriff der Strafverfolgung, weil sie schneller in ein anderes Land umziehen, als Gerichte für die Erstellung eines Durchsuchungsbefehls benötigen.

Sicherheit wird auf absehbare Zeit ein wichtiges Fokus-Thema in der Softwareentwicklung bleiben. Angriffe auf IT Systeme werden genauso wenig verschwinden wie die klassische Kriminalität, solange es ein lohnenswertes Ziel gibt. Auch im Jahre 2012 gibt es noch „echte“ Banküberfälle, die mit oder ohne Waffengewalt Banknoten aus einem Gebäude entwenden - entgegen anderslautender Prognosen zahlreicher IT Sicherheitsexperten.

Die Softwareindustrie entwickelt sich stetig von einem reaktiven („Patchen“ von Sicherheitslücken) zu einem proaktiven (Sicherheit als integraler Bestandteil des Softwareentwicklungsprozesses) Ansatz weiter. Dies erfolgt weitgehend aus der Erkenntnis, dass Sicherheit für ein Bestehen am Markt ein notwendiger Faktor ist, und nicht aus dem Zwang einer Regulierung oder Zertifizierung heraus.

Letztendlich müssen alle Beteiligten zu der Erkenntnis kommen, dass Sicherheit ihren Preis hat - wer seine Produkte vor staatlich motivierten „Cyberwar" Angriffen schützen will, muss mehr in Sicherheit investieren, als beispielsweise der Hersteller einer Bildbearbeitungssoftware für den Heimeinsatz.

Das vorliegende Heft zeigt einen aktuellen Querschnitt zu Sicherheit im Softwareentwicklungsprozess, beleuchtet die Bedrohungslage und gibt Einblicke von Experten zur Qualität der Softwareprodukte. Auf der ISSE 2012 in Brüssel soll dieses Wissen auch Experten aus vielen Ländern erreichen. Deshalb erscheinen die Schwerpunktbeiträge überwiegend in englischer Sprache.

Auch 2012 wird wieder das „Jahr der Hacks“ bleiben - genauso wie das führende Experten vor einem Jahr bereits über das vergangene Jahr 2010 (man erinnere sich an Stuxnet) gesagt hatten. Über eine absehbare Zeit werden sich am Markt Softwareunternehmen behaupten, die Sicherheit ernst nehmen und durch Qualität und eine geringere Zahl von Sicherheitslücken überzeugen können.

\section{Gunter Bitz}

